\title{
Improvement of Atopic Dermatitis by Synbiotic Baths
}

\author{
Matthias Noll *D, Michael Jäger ${ }^{1}$, Leonie Lux, Christian Buettner ${ }^{(D)}$ and Michaela Axt-Gadermann \\ Institute for Bioanalysis, Department of Applied Sciences, Coburg University of Applied Sciences and Arts, \\ 96450 Coburg, Germany; michael.jaeger93@gmx.de (M.J.); leonie.lux@behrens-pm.de (L.L.); \\ Christian.Buettner@hs-coburg.de (C.B.); michaela.axt-gadermann@hs-coburg.de (M.A.-G.) \\ * Correspondence: matthias.noll@hs-coburg.de; Tel.: +49-9561-317-645
}

Citation: Noll, M.; Jäger, M.; Lux, L.; Buettner, C.; Axt-Gadermann, M. Improvement of Atopic Dermatitis by Synbiotic Baths. Microorganisms 2021, 9, 527. https://doi.org/10.3390/ microorganisms 9030527

Academic Editor: Holger Brüggemann

Received: 1 February 2021

Accepted: 27 February 2021

Published: 4 March 2021

Publisher's Note: MDPI stays neutral with regard to jurisdictional claims in published maps and institutional affiliations.

Copyright: (c) 2021 by the authors. Licensee MDPI, Basel, Switzerland. This article is an open access article distributed under the terms and conditions of the Creative Commons Attribution (CC BY) license (https:/ / creativecommons.org/licenses/by/ $4.0 /)$.

\begin{abstract}
Atopic dermatitis (AD) is a widespread chronic inflammatory dermatologic disorder. This randomized, double-blind study aims to evaluate the effect of synbiotic baths with a defined mixture of six viable lactic acid bacteria (LAB) and prebiotics, without bacteria and prebiotics and placebo baths without prebiotics and bacteria to treat AD patients over a period of 14 days. Therefore, AD patients were randomly assigned into three groups using synbiotic $(n=7)$, prebiotics $(n=8)$ or placebo baths $(n=7)$. Severity of AD was evaluated over time by using severity scoring of atopic dermatitis (SCORAD) and by patient questionnaires. In addition, microbiome on eczematous skin surface was sampled by swaps from each patient before the bath treatment, and after 9, 11 and 14 days of bath treatment. Thereafter, nucleic acids were extracted and the bacterial 16S rRNA gene was amplified via PCR for subsequent amplicon sequencing. Results showed a significantly reduced SCORAD over time of AD patients after daily synbiotic or prebiotic baths. Moreover, AD patients after daily synbiotic baths had a significantly improved pruritus and skin dryness and their bacterial microbiome was enriched by LAB. Taken together, a synbiotic bath is a promising topical skin application to alleviate AD.
\end{abstract}

Keywords: amplicon sequencing; atopic dermatitis; bacterial 16 S rRNA gene; co-occurrence networks; human skin microbiome; severity scoring of atopic dermatitis; staphylococcus

\section{Introduction}

Atopic dermatitis (AD) is one of the most common dermatologic disorders affecting up to $20 \%$ of children as well as 1 to $3 \%$ of adults worldwide [1]. Pathogenesis is impacted by a multitude of genetic and environmental factors such as skin barrier dysfunction [2], abnormal protein and enzyme processing [3,4], impairment of tight junctions [5], impairment of the inflammatory cascade [6], dysbiosis of the skin microbiome [7] and exposure to irritant or proven allergic substances [8]. Due to the multifactorial nature of AD, efficient and well-tolerated treatment without any severe side effects are still challenging.

Treatment of AD can be categorized into basic skin care, topical corticosteroids (TCSs) and calcineurin inhibitors (TCIs), phototherapy or balneo phototherapy and systemic therapies based on immunosuppressive therapies or biologic drugs. Basic skin care is characterized by the application of emollients in order to moisturize the skin, restore the natural epidermal barrier and prevent further water loss $[9,10]$. However, its efficacy in moderate and severe cases of AD is limited. TCSs are placed on the skin by ointments, creams, sprays and foams [11]. TCIs can be used to inhibit the pro inflammatory cytokine gene transcription [12]. Despite their obvious merits, TCSs and TCIs are often scrutinized due to adverse side effects, which include ocular diseases [13], corticophobia [14], cutaneous atrophy, telangiectasia, striae, steroid rosacea and perioral dermatitis, hypothalamic pituitary-adrenal axis suppression and skin infections [15]. Finally, phototherapy is based on narrowband ultraviolet light two to three times per week over a timeframe of up to 12 weeks. Due to limited efficacy, it is most often used as a supplemental treatment $[16,17]$. Current treatments focus on affected local areas, while systemic treatments have the abil- 
ity to affect the entire body. Systematic treatment methodologies can cause untargeted suppression or modulation of components of the immune system [18].

Recent research indicate a correlation between AD severity and species distribution inside the microbiome of affected skin areas [19]. Staphylococcus aureus superinfection in particular was consistently linked with AD severity [20,21]. Based on these findings, manipulation of the skin microbiome via pre- and probiotics was considered as a potential treatment option for patients suffering from AD. Prebiotics are non-digestible ingredients that beneficially affect the host by stimulating growth of selected range of microorganisms [22]. Probiotics are defined as viable microorganisms that confer a health benefit on the host, when they are applied in adequate amounts [23]. Combinations of prebiotics and probiotics are known as synbiotics. Some studies indicate a reduction of AD occurrence and severity after oral probiotic treatments in infants and adults by a systematic treatment $[24,25]$. However, other studies showed no significant effect on AD after oral consumption of probiotics $[26,27]$ indicating that studies including a great variety of patient anamneses to retrieve a generalized conclusions are still lacking. In turn, information of local application of synbiotics or prebiotics to treat skin of AD patients is still rare. Synbiotic baths as local AD treatment showed a significant improvement of the AD patients' quality of life (QoL) [28]. These findings however rely on scoring of atopic dermatitis (SCORAD) and QoL measurements only. While these techniques are valuable tools, which allow profound statements of $\mathrm{AD}$ severity, no conclusions of changes in the skin microbiome can be stated.

This double-blind study aims to investigate the changes in the skin microbiomes of $\mathrm{AD}$ patients after local treatment with synbiotic, prebiotic or placebo baths as well as their correlation with patients' SCORAD and QoL over time. To this end, these data were correlated with the bacterial microbiome, which was achieved by a 16S rRNA gene amplicon sequencing approach. Additionally, a co-occurrence network analysis of the amplicon sequencing data was performed according to Olesen et al. [29] in order to explorer bacterial co-presence and mutual exclusion patterns between specific bacterial operational taxonomic units (OTUs) and bath treatments.

\section{Materials and Methods}

\subsection{Study Design}

A double-blind study with $22 \mathrm{AD}$ patients with atopic eczema on hands, arms, feet or legs was conducted. AD patients with an age less than five, pregnancy, immunodeficiency and/or ongoing antibiotic treatment were excluded prior to the start of the study. AD patients stopped other topical or systematic treatment seven days prior to the start of this study. AD severity of each patient was assessed by a dermatologist, and thereafter patients with similar AD severity were randomly assigned to three different treatment groups, which take a daily bath with a synbiotic $(n=7)$, prebiotic $(n=8)$ or placebo bath $(n=7)$. The synbiotic bath consisted of the probiotic strains $1 \times 10^{9}$ Colony forming units (CFUs) $\mathrm{L}^{-1}$ Bifidobacterium breve (ATCC 15698), $1 \times 10^{9} \mathrm{CFUs} \mathrm{L}^{-1}$ Bifidobacterium animalis subsp.

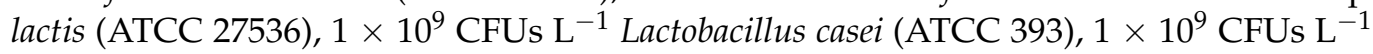
Lactobacillus gasseri (ATCC 33323), $1 \times 10^{9}$ CFUs L $^{-1}$ Lactobacillus plantarum (ATCC 14917) and $1 \times 10^{9} \mathrm{CFUs} \mathrm{L}^{-1}$ Lactobacillus rhamnosus (ATCC 53103), which were purchased from the American type culture collection and used after culturing as lyophilized powder in the synbiotic bath. As $0.5 \times 10^{9} \mathrm{CFUs}$ of each strain was available in one $\mathrm{g}$ (six strains resulted six $g$ including prebiotics), twelve gram per $\mathrm{L}$ were added and bath volume was approx. $4 \mathrm{~L}$ for legs and $3 \mathrm{~L}$ for arms. In addition, the synbiotic bath included $2.88 \mathrm{~g} \mathrm{~L}^{-1}$ maltodextrin, $6 \mathrm{~g} \mathrm{~L}^{-1}$ inulin, and $3 \mathrm{~g} \mathrm{~L}^{-1}$ apple pectin as prebiotics. The prebiotic bath contained only the prebiotics in the same concentration. Finally autoclaved sand was used as only ingredient for the placebo bath. Ingredients for each bath were separately added to hand-warm water and the skin surfaces with atopic eczema were bathed for ten minutes each day over a period of 14 days. After the bath remaining bath ingredients were dried on skin surface. The skin surface was documented by SCORAD as explained earlier [30], and sampled by 
cotton swaps (VWR International GmbH, Darmstadt, Germany) at the beginning of the experiment, and after nine, eleven and fourteen days of treatment. In addition, each AD patient assessed the own QoL by a questionnaire at the beginning of the experiment, after nine and fourteen days of treatment, which addressed the overall assessment, redness, pain, restriction, pruritus, lichenification and dryness of AD. Attending AD patients were asked to rate each parameter based on a scale ranging from one to ten with ten being the most severe by a questionnaire. To document any visible changes of the atopic eczema during the treatment period, photographs were taken at the beginning of the experiment, and after nine and fourteen days of treatment.

\subsection{Nucleic Acid Extraction}

Nucleic acids were extracted from the cotton swaps as described earlier [31] with minor modifications. Briefly, the head of the cotton swabs were cut with a sterile scissors and mixed with $0.2 \mathrm{~g}$ zirconium beads (Carl Roth $\mathrm{GmbH}$, Karlsruhe, Germany), $400 \mu \mathrm{L}$ cold TPM buffer (50 mM Tris $\mathrm{HCl} \mathrm{pH} 6.8 ; 1.7 \%(w / v)$ polyvinylpyrollidone $\mathrm{K} 25 ; 20 \mathrm{mM}$ $\mathrm{MgCl}_{2}$ ), $200 \mu \mathrm{L} \mathrm{NaPO}_{4}$ buffer (200mM NaPO 4 with pH 5.6) and $600 \mu \mathrm{L}$ SDS-phenol (all chemicals from Carl Roth). The mixture was vortexed and incubated for $10 \mathrm{~min}$ at $65{ }^{\circ} \mathrm{C}$. Thereafter, each sample was homogenized with a ball mill (FastPrep ${ }^{\circledR}$-24, Thermo Fisher Scientific, Waltham, MA, USA) for $60 \mathrm{~s}$ at $4.5 \mathrm{~m} \mathrm{~s}^{-1}$ and frozen for five minutes at $-80{ }^{\circ} \mathrm{C}$. Each sample was subsequently centrifuged for $15 \mathrm{~min}$ at $21.500 \times g$ and $4{ }^{\circ} \mathrm{C}$. Supernatant (approx. $800 \mu \mathrm{L}$ ) was mixed with the same volume of TPM buffer and centrifuged again $\left(21,500 \times g, 4^{\circ} \mathrm{C}, 15 \mathrm{~min}\right)$. Thereafter, $800 \mu \mathrm{L}$ of supernatant was mixed with the same volume of phenol-chloroform-isoamylalcohol (PCI, Carl Roth) and centrifuged once more $\left(21,500 \times g, 4^{\circ} \mathrm{C}, 15 \mathrm{~min}\right) .650 \mu \mathrm{L}$ supernatant was mixed with $1300 \mu \mathrm{L}$ PEG buffer $(30 \%$ polyethylene glycol 6000 in $1.6 \mathrm{M} \mathrm{NaCl}$-solution) and $2 \mu \mathrm{L}$ glycogen (VWR International $\mathrm{GmbH})$. The mixture was centrifuged for $45 \mathrm{~min}$ at $4{ }^{\circ} \mathrm{C}$ and $21,500 \times g$. The supernantant was discarded. The resulting nucleic acid pellet was washed two times with ice-cold $500 \mu \mathrm{L}$ $70 \%$ ethanol and dried at $37^{\circ} \mathrm{C}$. The pellet was then resuspended in $50 \mu \mathrm{L} 1 \times \mathrm{TE}$ buffer (Carl Roth). Quality and quantity of nucleic acids was measured with Thermo Scientific ${ }^{\mathrm{TM}}$ Multiskan ${ }^{\mathrm{TM}}$ GO Microplate Spectrophotometer (Thermo Fisher Scientific) as outlined by the manufacturer.

\subsection{PCR and Amplicon Sequencing}

$16 \mathrm{~S}$ rRNA gene was amplified from each of the 80 nucleic acid extracts using the $341 \mathrm{~F}$ (5'CCTACGGGNGGCWGCAG'3) and 785R (5'GACTACHVGGGTATCTAATCC'3) primer pair [32] as described earlier [33]. PCR amplicons were thereafter ligated to inline barcode sequences and subsequently 300 bp paired-end sequenced by Illumina Miseq V3 System (San Diego, CA, USA), which was carried out by LGC Genomics (LGC Genomics GmbH, Berlin, Germany). Sequence raw data were demultiplexed by using the Illumina bcl2fastq 2.17.1.14 software (Illumina, San Diego, CA, USA) and were subsequently sorted by reads of amplicon inline barcodes. Barcode sequences, adapters and primers were clipped from the sequence and forward and reverse reads were combined by using BBMerge 34.48 [34]. 16S rRNA gene sequences were pre-processed and operational taxonomic units (OTUs) were picked from the amplicons with Mothur 1.35.1 [35]. Sequences with ambiguous bases, with homopolymer stretches, short reads, chimera and with an average quality score below 33 were removed [35]. 16S rRNA gene sequences were aligned with the 16S Mothur-Silva SEED r119 reference database [35], and OTUs were picked by clustering at the $97 \%$ identity level and taxonomical classified against the Silva reference classification [36]. Two samples were discarded due to low sequencing read counts. Thereafter, singleton and doubleton OTUs were removed and a table with individual 937 OTUs was generated (Supplementary Table S3). The shannon diversity index, the simpson's diversity index and the Chao1 richness estimator were calculated for all 78 samples using the diversity and estimateR functions R-package vegan [37]. The data was visualized using the Origin 2019b program (Origin Lab Corporation, Northampton, MA, USA). 


\subsection{Statistics and Network Analyses}

Normal distribution of the data set was tested for the 10 most abundant OTUs over time by the Shapiro-Wilk test [38] using the software Origin 2019b (OriginLab Corporation, Northampton, MA, USA). Two-way ANOVA was used to test the effect of SCORAD, QoL parameters, bacterial diversity indices over time and over bath treatment and over the interaction of time and bath treatment. The significance threshold was defined as $p \leq 0.05$. Correspondence analysis was carried out with relative sequence read abundances as reported earlier [33].

Co-occurrence network analyses for each bath treatment were calculated by using cytoscape with the plugin CoNet $[39,40]$. OTU table of each treatment with absolute sequence counts were used, if OTUs were present in at least three AD patients of a treatment bath group. Network calculations were carried out with following methods: Pearson, Spearman correlation, Steinhaus similarity, Bray-Curtis and Kullback-Leibler dissimilarity. Minimal occurrence of observations for each set of replicates was set to at least $60 \%$ (i.e., 24,15 and 9 observations for the different networks). Threshold was set to 3000 top and bottom edges, so that each correlation and dissimilarity method contributed 3,000 positive and 3000 negative edges to the initial network. MinSupport was selected to be three, so only edges supported by at least three of the five methods were kept. The methodspecific $p$-values were computed by using the mean and standard deviation of the bootstrap distribution (100 iterations) as a parameter of the normal distribution. Method-specific $p$-values were then merged using the method of Brown [41]. Only edges with $p<0.05$ were kept after multiple-testing correction of Benjamini and Hochberg [42]. In addition, the difference between all co-occurrence networks from synbiotic, prebiotic and placebo bath treatments was also calculated with the same plugin CoNet using the merge difference function. The resulting merged co-occurrence network enabled the examination of changes in network compositions between bath treatments. Nodes of the final networks were assigned to modules by using GLay community algorithm [43].

Finally, within-module connectivity (z) and among-module connectivity (Pi) were calculated as described by Guimera and Amaral [44] with an automated in-house excel sheet. Peripheral nodes (specialists) were defined by $\mathrm{z} \leq 2.5$ and $\mathrm{Pi} \leq 0.62$, connectors by $\mathrm{z} \leq 2.5$ and $\mathrm{Pi}>0.62$, module hubs by $\mathrm{z}>2.5$ and $\mathrm{Pi} \leq 0.62$ and network hubs by $\mathrm{z}>2.5$ and $\mathrm{Pi}>0.62$ as introduced earlier [29].

\section{Results}

\subsection{Synbiotic Baths Improved SCORAD and QoL}

The SCORAD of AD patients after 14 days of a daily synbiotic or prebiotic baths decreased significantly (Figure 1). Moreover, SCORAD of AD patients of synbiotic bath improved significantly better compared to the other bath treatments over time. In contrast, the SCORAD of AD patients after a daily placebo bath did not change over time.

In addition, the $\mathrm{AD}$ patients evaluated their skin appearance and their QoL. After all synbiotic baths, AD patients recognized significantly reduced pruritus and skin dryness over time (Table 1). In addition, overall assessment, restriction, redness, pain and lichenification improved over time for $\mathrm{AD}$ patients after synbiotic or prebiotic baths but not for AD patients after placebo baths. Moreover, photographs of the AD sides of patients after synbiotic or prebiotic baths showed improvements of the skin appearance (Supplementary Figure S1). 


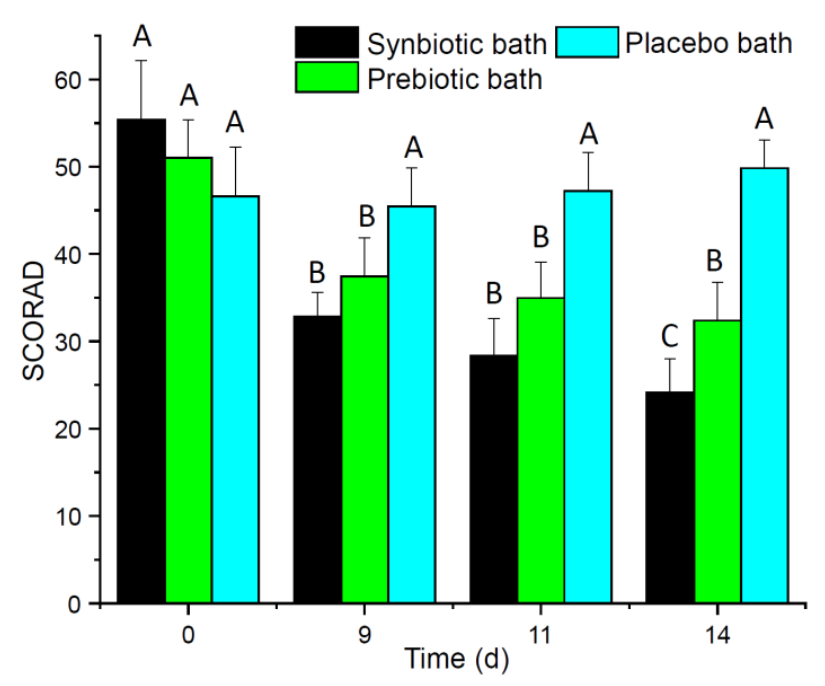

Figure 1. Severity scoring of atopic dermatitis (SCORAD) of atopic dermatitis (AD) patients after daily synbiotic (black bar, $n=7$ ), prebiotic (green bar, $n=8$ ) or placebo bath (cyan bar, $n=7$ ) over time. Error bars indicate standard deviation. Different letters above bars within panels indicate significant differences $(p<0.05)$ according to two-way analysis of variance (ANOVA).

Table 1. Two-way analysis of variance (ANOVA) of quality of life (QoL) parameters and severity scoring of atopic dermatitis (SCORAD) from AD patients after 14 days of daily synbiotic, prebiotic or placebo bath treatment. Significance of bath treatments, time or the interaction of bath treatments and time is highlighted in bold $(p<0.05)$. DF, degree of freedom.

\begin{tabular}{|c|c|c|c|c|c|c|}
\hline Parameter & DF & F Value & $p$ Value & DF & F Value & $p$ Value \\
\hline & \multicolumn{3}{|c|}{ Overall assessment } & \multicolumn{3}{|c|}{ Pain } \\
\hline Time & 2 & 282.99 & 0.07 & 2 & 116.54 & 0.32 \\
\hline Bath treatment & 2 & 559.11 & $<0.01$ & 2 & 522.90 & $<0.01$ \\
\hline Bath treatment $\times$ Time & 4 & 0.77 & 0.55 & 4 & 0.27 & 0.90 \\
\hline & \multicolumn{3}{|c|}{ Restriction } & \multicolumn{3}{|c|}{ Lichenification } \\
\hline Time & 2 & 15.76 & 0.22 & 2 & 183.79 & 0.17 \\
\hline Bath treatment & 2 & 688.58 & $<0.01$ & 2 & 147.13 & $<0.01$ \\
\hline \multirow[t]{2}{*}{ Bath treatment $\times$ Time } & 4 & 0.25 & 0.91 & 4 & 0.66 & 0.62 \\
\hline & \multicolumn{3}{|c|}{ Pruritus } & \multicolumn{3}{|c|}{ Dryness } \\
\hline Time & 2 & 416.52 & 0.02 & 2 & 89.01 & $<0.01$ \\
\hline Bath treatment & 2 & 759.94 & $<0.01$ & 2 & 921.14 & $<0.01$ \\
\hline \multirow[t]{2}{*}{ Bath treatment $\times$ Time } & 4 & 0.92 & 0.46 & 4 & 136.97 & 0.26 \\
\hline & & \multicolumn{2}{|c|}{ Redness } & \multicolumn{3}{|c|}{ SCORAD } \\
\hline Time & 2 & 0.92 & 0.40 & 2 & 889.67 & $<0.01$ \\
\hline Bath treatment & 2 & 986.83 & $<0.01$ & 2 & 1060.98 & $<0.01$ \\
\hline Bath treatment $\times$ Time & 4 & 0.68 & 0.61 & 4 & 408.78 & $<0.01$ \\
\hline
\end{tabular}

\subsection{Composition of the Skin Microbiome Was Shifted}

The composition of the bacterial microbiome shifted over time and was more similar to each other from $\mathrm{AD}$ patients after synbiotic bath treatment compared to prebiotic or placebo bath treatment as these both treatments revealed a higher compositional variance (Figure 2). Members of the genus Staphylococcus were the most dominant taxa of the skin microbiomes of $\mathrm{AD}$ patients, which covered approx. one-third to two-thirds of the relative sequence read abundance of all samples (Supplementary Figure S2). AD patients treated with synbiotic baths exhibited the highest relative sequence read abundance of members of the genus Staphylococcus before the bath treatment, which is in accordance to the severity reflected by the SCORAD (Figure 1). Although the SCORAD significantly decreased, the sequence read abundance of members of the genus Staphylococcus did not significantly alter over time (Supplementary Table S3). 

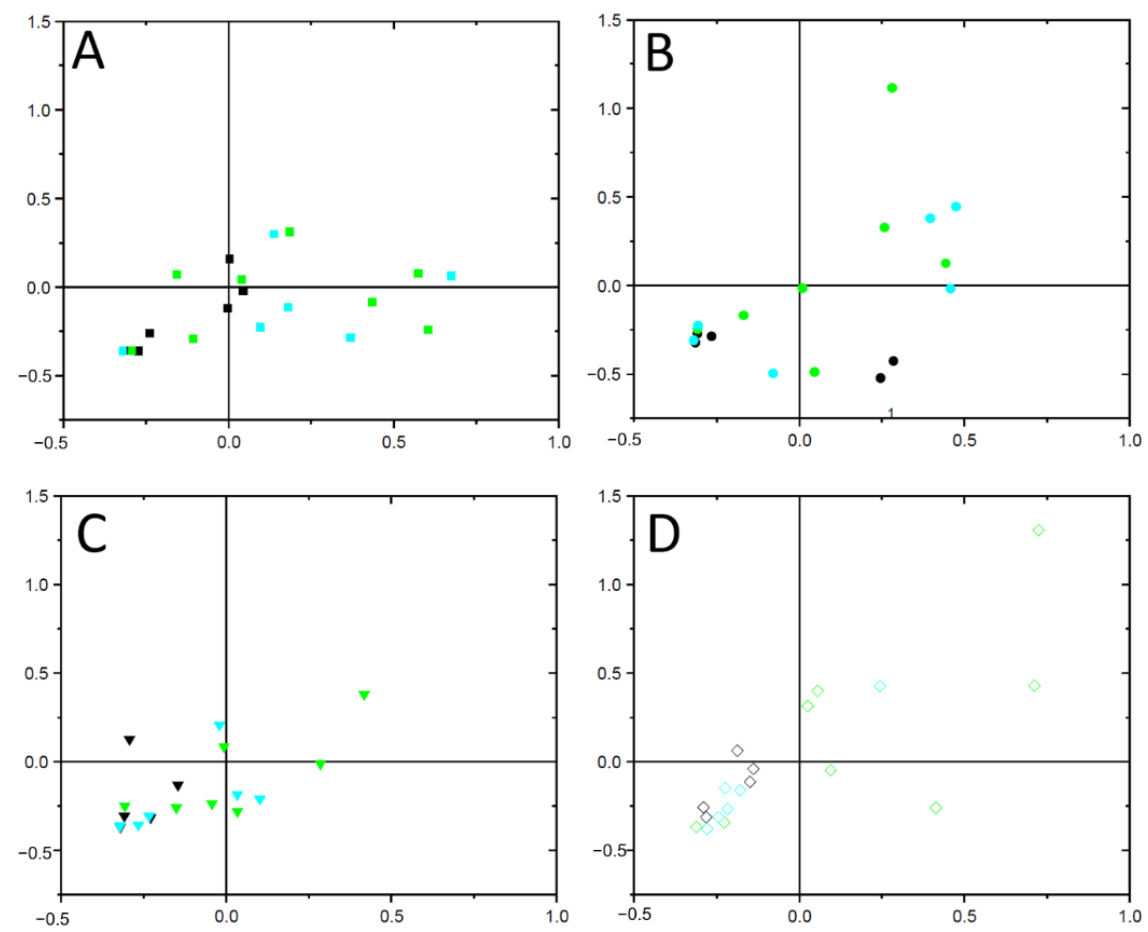

Figure 2. Correspondence analysis of the bacterial skin microbiome retrieved from AD patients after daily synbiotic (black), prebiotic (green) or placebo bath (cyan) before start $(\mathbf{A}, \mathbf{\square})$, and after $9(\mathbf{B}, \bullet$ ), $11(\mathbf{C}, \mathbf{\nabla})$ and 14 days of bath treatment $(\mathbf{D}, \diamond)$. The eigenvalues of the 1 st and 2 nd axes were $\lambda_{1}=0.53$ and $\lambda_{2}=0.48$.

Members of the genera Enhydrobacter, Corynebacterium, Acinetobacter, Micrococcus, Escherichia and Paracoccus were also abundant (Supplementary Figure S2). Relative sequence read abundances of members of the genera Lactobacillus and Bifidobacterium increased from none to $8 \%$ and $6 \%$, respectively, over time only by $\mathrm{AD}$ patients treated with synbiotic baths, which contained these species (Supplementary Figure S3). Therefore, the viable microorganisms as part of the synbiotic bath ingredients became part of the bacterial skin microbiome already after nine days of bath treatment, which also affected the bacterial microbiome composition (Figure 2). AD patients treated with prebiotic baths were characterized by relative sequence read abundances of members of the genera Micrococcus and Paracoccus (Supplementary Figure S2). In turn, AD patients treated with placebo baths showed an increase in relative sequence abundances of members of the genera Staphylococcus, Paracoccus and Enhydrobacter and a decrease of Corynebacterium over time (Supplementary Figure S2). The diversity indices Shannon and Simpson's and Chao1 richness estimator did not alter significantly over time in any bath treatment (Supplementary Table S1).

\subsection{Bacterial Co-Occurrence Networks of AD Patients Differed}

Three independent co-occurrence network analyses were individually calculated for AD patients after daily synbiotic, prebiotic or placebo bath treatments, which consisted of 2274, 1990 and 1960 significant interactions (edges) between 241, 338 and 338 different OTUs (nodes), and six, 13 and ten distinct modules, respectively. Members of the genus Micrococcus were identified as a network hub of AD patients after synbiotic bath treatment (Figure 3A). Network of AD patients after a prebiotic bath showed a non-cultured genus of the order Obscuribacterales as a module hub, and members of the genera Caulobacter, Sphingopyxis, Aquabacterium and Chthoniobacter as module hubs (Figure 3B, Table 2). Network of AD patients after a placebo bath showed the highest connectivity among the three networks, with four module hubs and 15 connectors (Figure 3C, Table 2). Notably, no significant interactions with the dominant members of the genus Staphylococcus were identified in any network analyses (Supplementary Figure S3). 

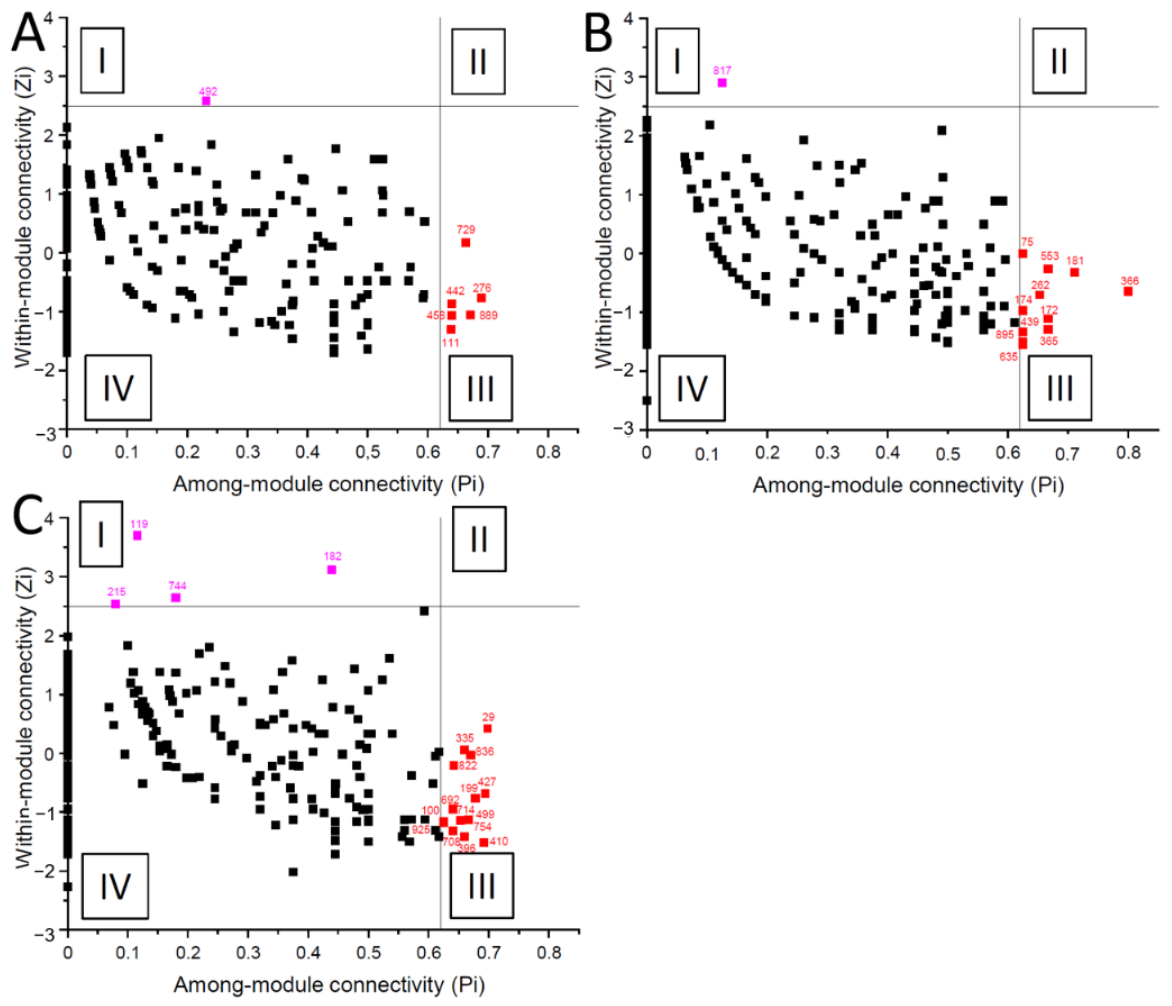

Figure 3. Network roles of each OTU of the bacterial skin microbiome retrieved from AD patients after daily synbiotic (A), prebiotic (B) or placebo bath treatment (C). Each OTU was categorized into module hubs (I), network hubs (II) connectors (III) or peripherals (IV) according to Olesen et al. (2007) [29]. OTU details of I-III can be found in Table 2 and OTU details of IV can be found in Supplementary Table S2.

In addition, a merged network based on differences between these three co-occurrence networks was generated, which consisted of 1817 interactions of 337 OTUs between each bath treatment group (Supplementary Figure S4). Therefore, substantial differences in interactions patterns between groups were illustrated. Members of the genus Acidibacter exhibited more interactions in the microbiomes of AD patients with placebo baths compared to AD patients that used synbiotic or prebiotic baths (Supplementary Table S2). Members of the genus Propionibacterium, had a more prominent topological role for AD patients after a prebiotic bath.

Table 2. Taxonomic classification and topological role of OTUs of the three co-occurrence networks of the bacterial skin microbiome retrieved from AD patients after 14 days of daily synbiotic, prebiotic or placebo bath treatment. OTUs of the network were assigned to connectors, module hubs or network hubs according to Olesen et al. (2007) [29] (see also Figure 3).

\begin{tabular}{cccc}
\hline Treatment & OTU Number & Module & Taxon \\
\hline & 889 & connector & Acidibacter \\
& 442 & connector & Lachnoanaerobaculum \\
& 492 & module hub & Micrococcus \\
Synbiotic bath & 111 & connector & Brevibacterium \\
& 729 & connector & Conexibacter \\
& 276 & connector & Dermabacter \\
& 458 & connector & Leptotrichia \\
& 456 & connector & Pediococus \\
& 696 & connector & Rickettsiales \\
\hline
\end{tabular}


Table 2. Cont.

\begin{tabular}{|c|c|c|c|}
\hline Treatment & OTU Number & Module & Taxon \\
\hline \multirow{12}{*}{ Prebiotic bath } & 365 & connector & Bergeyella \\
\hline & 366 & connector & Chryseobacterium \\
\hline & 75 & connector & Geobacillus \\
\hline & 172 & connector & Alloiococcus \\
\hline & 181 & connector & Brevundimonas \\
\hline & 174 & connector & Carnobacterium \\
\hline & 895 & connector & Dialister \\
\hline & 439 & connector & Howardella \\
\hline & 553 & connector & Marinomonas \\
\hline & 817 & module hub & Obscuribacterales \\
\hline & 262 & connector & Ohtaekwangia \\
\hline & 635 & connector & Propionibacterium \\
\hline \multirow{17}{*}{ Placebo bath } & 499 & connector & Actinoplanes \\
\hline & 119 & module hub & Aquabacterium \\
\hline & 215 & module hub & Chthoniobacter \\
\hline & 410 & connector & Janibacter \\
\hline & 754 & connector & Jeotgalicoccus \\
\hline & 29 & connector & Ilumatobacter \\
\hline & 836 & connector & Acidobacteria \\
\hline & 100 & connector & Bifidobacteriaceae \\
\hline & 182 & module hub & Caulobacter \\
\hline & 396 & connector & Hydrogenophilus \\
\hline & 335 & connector & Peptoniphilus \\
\hline & 925 & connector & Rhodanobacter \\
\hline & 692 & connector & Rhodospirillales \\
\hline & 708 & connector & Ruminococcaceae \\
\hline & 822 & connector & Saccharimonadales \\
\hline & 744 & module hub & Sphingopyxis \\
\hline & 714 & connector & Subdoligranulum \\
\hline
\end{tabular}

\section{Discussion}

The majority of AD patients had a long history of topical and systematic treatments to treat their AD. As AD patients has to stop their previous treatment seven days prior this study a typical rebound phenomenon was observed, which has been described before [45]. This rebound phenomenon affected the early phase of each bath treatment as the SCORAD after three and seven days was not significantly different to the initial SCORAD. Therefore, we chose 9,11 and 14 days of each bath treatment for in depth microbiome analyses.

AD severity is very often linked to the abundance of $S$. aureus in the AD skin microbiome [46], and also AD patients in this study showed a high relative sequence read abundances of members of the genus Staphylococcus (Supplementary Figure S2), which was correlated to the SCORAD (Figure 1). This high relative sequence read abundances in the microbiome is in contrast to microbiomes of non-AD skin patient, which reported lower sequence read abundances for Staphylococcus of 20 to $40 \%$ [47]. In addition, the other abundant genera Enhydrobacter, Corynebacterium, Acinetobacter, Micrococcus and Streptococcus commonly also occur as part of the human skin microbiome [47]. Therefore AD patients of this study showed a common skin microbiome with increased relative abundance of members of the genus Staphylococcus, which has been reported frequently before [20,21]. As the 16S rRNA gene sequence approach of the V3-V4 regions has a low phylogenetic resolution on species level for the skin microbiome [48], this study cannot differentiate shifts between S. aureus and other Staphylococcus spp. such as S. epidermidis or temporal/treatment specific shifts within the genus Staphylococcus.

In addition, the OTUs affiliated to the genus Staphylococcus showed no significant interactions (edges) in any bath treatment of the co-occurrence networks as these OTUs were always present and which do not reflect shifts in co-presence or mutual exclusions. 
Aside from members of the genus Staphylococcus relative sequence read abundance of AD patients after daily prebiotic and synbiotic bath treatment was shown to impact the composition of the microbiomes (Figure 2) as well as interaction patterns between members of the respective bacterial communities (Figure 3). Future research should also address the effect of probiotic treatment without prebiotics.

AD patients after daily synbiotic and prebiotic baths had a significantly decreased SCORAD and clearly visible improvement of their skin complexion and all QoL parameters (Table 1). Therefore, the application of the prebiotic components such as maltodextrin, inulin and apple pectin were beneficial for skin appearance, which has been shown for inulin also recently in another study [49]. However, the other prebiotic components were so far not studied in detail but may serve as nutrient for the beneficial skin microbiome. Moreover, skin dryness and pruritus was significantly better for AD patients after daily synbiotic baths. The bacterial microbiome showed an increase of the relative sequence reads of the genera Lactobacillus and Bifidobacterium, which were viable ingredient of the bath, only for AD patients treated with a synbiotic bath. The four strains from the genus Lactobacillus were described to modulate the host immune system through cytokine gene expression and to stimulate the phagocytosis by peripheral blood leucocytes [50]. In addition, both Bifidobacterium and all four Lactobacillus strains of the bath ingredient are potential bacteriocin producers and therefore able to limit the growth of opportunistic pathogens [51-55]. Moreover member of the genus Bifidobacterium can stimulate human immune function [56]. Furthermore, relative sequence read abundance of Corynebacterium decreased in the microbiome after synbiotic baths. Members of the genus Corynebacterium were associated with AD severity in the past [57]. Interestingly, OTUs affiliated to the order Corynebacteriales exhibited less interactions with other members of the bacterial microbiome with increasing treatment time (Supplementary Table S2) indicating a decreasing importance in the co-occurrence network. Moreover, Kwaszewska and colleagues showed a synbiotic relationship between $S$. aureus and members of the genus Corynebacterium due to a lack of proteinase activity [58]. Members of the genus Brevibacterium had also a decreasing topological role over time, and were reported as human pathogens capable to cause skin infections [59]. In turn, members of the genera Acidibacter and Micrococcus were described as module hubs from $\mathrm{AD}$ patients after synbiotic baths. While members of the genus Acidibacter were rarely found on human skin environments, Micrococcus is in top ten of the most frequent species of healthy human skin microbiomes [47] and is present only in low abundances in the skin microbiome of AD patients [60].

\section{Conclusions}

In conclusion, the treatment of $\mathrm{AD}$ patients after a daily synbiotic and to a lesser extend with a daily prebiotic bath decreased significantly the SCORAD and progressively improved QoL parameters. In addition, skin microbiome of AD patients after a synbiotic bath were colonized by strains from the probiotic bath. These strains have the generally recognized as safe (GRAS) status as probiotics and our results indicate that these strains are also harmless for skin applications. Taken together, this proof-of-concept study showed that a daily synbiotic bath, which included safe lactic acid bacteria strains, is a promising topical skin application to alleviate AD. Upcoming research should include more patients and address shifts in the host-microbe interaction of $\mathrm{AD}$ patients treated with synbiotic baths.

Supplementary Materials: The following are available online at https://www.mdpi.com/2076-2 607/9/3/527/s1, Figure S1: Changes in skin appearance from AD patients after daily synbiotic baths (A), prebiotic baths (B) or placebo baths (C) over time (start of incubation $=1$; after 14 days of bath treatment $=2)$; Figure S2: Relative sequence read abundances of the bacterial skin microbiome retrieved from $\mathrm{AD}$ patients after daily synbiotic (A), prebiotic (B) or placebo bath (C) over time; Figure S3: Relative sequence read abundances of members of the genera Bifidobacterium (A) and Lactobacillus (B) as part of the bacterial skin microbiome retrieved from AD patients after daily synbiotic, prebiotic or placebo bath over time; Figure S4: Co-occurrence network from the differences 
of the bacterial skin microbiome networks retrieved from AD patients after daily synbiotic baths, prebiotic baths or placebo baths. In total, ten different modules were found. Further details can be found in the material and methods section in the main document; Table S1: Two-way analysis of variance (ANOVA) of diversity indices from the bacterial skin microbiome derived from AD patients that attended synbiotic, prebiotic or placebo baths over time. Significance of bath treatment, time or the interaction of bath treatment and time is highlighted in bold $(p<0.05)$. DF, degree of freedom; Table S2: Topological role of OTUs in co-occurrence networks derived from AD patients that attended synbiotic, prebiotic or placebo baths and a merged network with the differences in the topological role between all three bath treatments; Table S3: Overview of the bacterial community composition derived from AD patients that attended synbiotic, prebiotic or placebo baths. All OTUs are summarized up to genus level.

Author Contributions: Conceptualization, M.N. and M.A.-G.; methodology, L.L.; validation, M.N., M.J. and L.L.; formal analysis, L.L. and M.J.; investigation, L.L.; resources, M.N.; data curation, C.B.; writing-original draft preparation, M.J. and M.N.; writing—review and editing, M.N.; visualization, M.J.; supervision, M.N.; project administration, M.N. All authors have read and agreed to the published version of the manuscript.

Funding: This research received no external funding.

Institutional Review Board Statement: The bath treatments for patients suffering from AD was approved by the ethic commission Coburg (Germany) in their 24th meeting in June 2018. The study was conducted according to the guidelines of the Declaration of Helsinki, and all official requirements were implemented in the study design and patient communication were performed in accordance with the ethic guidelines and regulations. Prior to subject's participation in this study, the written informed consent form was signed and personally dated by each patient.

Informed Consent Statement: Informed consent was obtained from all subjects involved in the study.

Data Availability Statement: The bacterial 16S rRNA gene sequences were deposited in the NCBI nucleotide sequence databases under accession PRJNA664092.

Acknowledgments: We thank all AD patients for the willingness to join this study. In addition, we thank Katharina Trunzer for technical assistance.

Conflicts of Interest: The authors declare no conflict of interest.

\section{References}

1. Mayba, J.N.; Gooderham, M.J. Review of atopic dermatitis and topical therapies. J. Cutan. Med. Surg. 2017, 21, 227-236. [CrossRef]

2. Van Smeden, J.; Bouwstra, J.A. Stratum Corneum Lipids: Their role for the skin barrier function in healthy subjects and atopic dermatitis patients. Curr. Probl. Dermatol. 2016, 49, 8-26. [CrossRef] [PubMed]

3. McAleer, M.A.; Jakasa, I.; Raj, N.; O’Donnell, C.P.F.; Lane, M.E.; Rawlings, A.V.; Voegeli, R.; McLean, W.H.I.; Kezic, S.; Irvine, A.D. Early-life regional and temporal variation in filaggrin-derived natural moisturizing factor, filaggrin-processing enzyme activity, corneocyte phenotypes and plasmin activity: Implications for atopic dermatitis. Br. J. Dermatol. 2018, 179, 431-441. [CrossRef]

4. Naeem, A.S.; Tommasi, C.; Cole, C.; Brown, S.J.; Zhu, Y.; Way, B.; Willis Owen, S.A.G.; Moffatt, M.; Cookson, W.O.; Harper, J.I.; et al. A mechanistic target of rapamycin complex 1/2 (mTORC1)/V-Akt murine thymoma viral oncogene homolog 1 (AKT1)/cathepsin H axis controls filaggrin expression and processing in skin, a novel mechanism for skin barrier disruption in patients with atopic dermatitis. J. Allergy Clin. Immunol. 2017, 139, 1228-1241. [CrossRef]

5. Yuki, T.; Tobiishi, M.; Kusaka-Kikushima, A.; Ota, Y.; Tokura, Y. Impaired tight junctions in atopic dermatitis skin and in a skin-equivalent model treated with interleukin-17. PLoS ONE 2016, 11, e0161759. [CrossRef] [PubMed]

6. Furue, M.; Chiba, T.; Tsuji, G.; Ulzii, D.; Kido-Nakahara, M.; Nakahara, T.; Kadono, T. Atopic dermatitis: Immune deviation, barrier dysfunction, IgE autoreactivity and new therapies. Allergol. Int. 2017, 66, 398-403. [CrossRef]

7. Seite, S.; Flores, G.E.; Henley, J.B.; Martin, R.; Zelenkova, H.; Aguilar, L.; Fierer, N. Microbiome of affected and unaffected skin of patients with atopic dermatitis before and after emollient treatment. J. Drugs Dermatol. 2014, 13, 1365-1372. [PubMed]

8. Kim, K. Influences of environmental chemicals on atopic dermatitis. Toxicol. Res. 2015, 31, 89-96. [CrossRef] [PubMed]

9. Simpson, E.L.; Chalmers, J.R.; Hanifin, J.M.; Thomas, K.S.; Cork, M.J.; McLean, W.H.I.; Brown, S.J.; Chen, Z.; Chen, Y.; Williams, H.C. Emollient enhancement of the skin barrier from birth offers effective atopic dermatitis prevention. J. Allergy Clin. Immunol. 2014, 134, 818-823. [CrossRef]

10. Tiplica, G.S.; Boralevi, F.; Konno, P.; Malinauskiene, L.; Kaszuba, A.; Laurens, C.; Saint-Aroman, M.; Delarue, A. The regular use of an emollient improves symptoms of atopic dermatitis in children: A randomized controlled study. J. Eur. Acad. Dermatol. Venereol. 2018, 32, 1180-1187. [CrossRef] 
11. Del Rosso, J.; Friedlander, S.F. Corticosteroids: Options in the era of steroid-sparing therapy. J. Am. Acad. Dermatol. 2005, 53, S50-S58. [CrossRef] [PubMed]

12. Bornhövd, E.; Burgdorf, W.H.; Wollenberg, A. Macrolactam immunomodulators for topical treatment of inflammatory skin diseases. J. Am. Acad. Dermatol. 2001, 45, 736-743. [CrossRef]

13. Daniel, B.S.; Orchard, D. Ocular side-effects of topical corticosteroids: What a dermatologist needs to know. Australas. J. Dermatol. 2015, 56, 164-169. [CrossRef] [PubMed]

14. Chiricozzi, A.; Comberiati, P.; D'Auria, E.; Zuccotti, G.; Peroni, D.G. Topical corticosteroids for pediatric atopic dermatitis: Thoughtful tips for practice. Pharmacol. Res. 2020, 158, 104878. [CrossRef]

15. Spada, F.; Barnes, T.M.; Greive, K.A. Comparative safety and efficacy of topical mometasone furoate with other topical corticosteroids. Australas. J. Dermatol. 2018, 59, e168-e174. [CrossRef]

16. George, S.A.; Bilsland, D.J.; Johnson, B.E.; Ferguson, J. Narrow-band (TL-01) UVB air-conditioned phototherapy for chronic severe adult atopic dermatitis. Br. J. Dermatol. 1993, 128, 49-56. [CrossRef]

17. Reynolds, N.J.; Franklin, V.; Gray, J.C.; Diffey, B.L.; Farr, P.M. Narrow-band ultraviolet B and broad-band ultraviolet A phototherapy in adult atopic eczema: A randomised controlled trial. Lancet 2001, 357, 2012-2016. [CrossRef]

18. Simpson, E.L.; Bruin-Weller, M.; Flohr, C.; Ardern-Jones, M.R.; Barbarot, S.; Deleuran, M.; Bieber, T.; Vestergaard, C.; Brown, S.J.; Cork, M.J.; et al. When does atopic dermatitis warrant systemic therapy? Recommendations from an expert panel of the International Eczema Council. J. Am. Acad. Dermatol. 2017, 77, 623-633. [CrossRef] [PubMed]

19. Leung, D.Y.M. The microbiome and allergic diseases: A struggle between good and bad microbes. Ann. Allergy Asthma Immunol. 2019, 122, 231-232. [CrossRef]

20. Huang, J.T.; Abrams, M.; Tlougan, B.; Rademaker, A.; Paller, A.S. Treatment of Staphylococcus aureus colonization in atopic dermatitis decreases disease severity. Pediatrics 2009, 123, e808-14. [CrossRef]

21. Nakatsuji, T.; Chen, T.H.; Narala, S.; Chun, K.A.; Two, A.M.; Yun, T.; Shafiq, F.; Kotol, P.F.; Bouslimani, A.; Melnik, A.V.; et al. Antimicrobials from human skin commensal bacteria protect against Staphylococcus aureus and are deficient in atopic dermatitis. Sci. Transl. Med. 2017, 9, eaah4680. [CrossRef]

22. Gibson, G.R.; Roberfroid, M.B. Dietary modulation of the human colonic microbiota: Introducing the concept of prebiotics. J. Nutr. 1995, 125, 1401-1412. [CrossRef]

23. Hill, C.; Guarner, F.; Reid, G.; Gibson, G.R.; Merenstein, D.J.; Pot, B.; Morelli, L.; Canani, R.B.; Flint, H.J.; Salminen, S.; et al. Expert consensus document. The international scientific association for probiotics and prebiotics consensus statement on the scope and appropriate use of the term probiotic. Nat. Rev. Gastroenterol. Hepatol. 2014, 11, 506-514. [CrossRef]

24. Isolauri, E.; Arvola, T.; Sütas, Y.; Moilanen, E.; Salminen, S. Probiotics in the management of atopic eczema. Clin. Exp. Allergy 2000, 30, 1604-1610. [CrossRef] [PubMed]

25. Rautava, S.; Kalliomäki, M.; Isolauri, E. Probiotics during pregnancy and breast-feeding might confer immunomodulatory protection against atopic disease in the infant. J. Allergy Clin. Immunol. 2002, 109, 119-121. [CrossRef] [PubMed]

26. Lee, J.; Seto, D.; Bielory, L. Meta-analysis of clinical trials of probiotics for prevention and treatment of pediatric atopic dermatitis. J. Allergy Clin. Immunol. 2008, 121, 116-121.e11. [CrossRef] [PubMed]

27. Cuello-Garcia, C.A.; Brożek, J.L.; Fiocchi, A.; Pawankar, R.; Yepes-Nuñez, J.J.; Terracciano, L.; Gandhi, S.; Agarwal, A.; Zhang, Y.; Schünemann, H.J. Probiotics for the prevention of allergy: A systematic review and meta-analysis of randomized controlled trials. J. Allergy Clin. Immunol. 2015, 136, 952-961. [CrossRef] [PubMed]

28. Axt-Gadermann, M. Signifikante Verbesserung des atopischen Ekzems durch Therapie mit synbiotischem Badezusatz. Akt Dermatol 2018, 44, 366-373. [CrossRef]

29. Olesen, J.M.; Bascompte, J.; Dupont, Y.L.; Jordano, P. The modularity of pollination networks. Proc. Natl. Acad. Sci. USA 2007, 104, 19891-19896. [CrossRef] [PubMed]

30. Stalder, J.F.; Taieb, A.; Atherton, D.J.; Bieber, P. Severity Scoring of Atopic Dermatitis: The SCORAD Index. Consensus Report of the European Task Force on Atopic Dermatitis. Dermatology (Basel) 1993, 186, 23-31. [CrossRef]

31. Noll, M.; Matthies, D.; Frenzel, P.; Derakshani, M.; Liesack, W. Succession of bacterial community structure and diversity in a paddy soil oxygen gradient. Environ. Microbiol. 2005, 7, 382-395. [CrossRef] [PubMed]

32. Thijs, S.; Beeck, M.o.d.; Beckers, B.; Truyens, S.; Stevens, V.; van Hamme, J.D.; Weyens, N.; Vangronsveld, J. Comparative evaluation of four Bacteria-specific primer pairs for 16S rRNA gene surveys. Front. Microbiol. 2017, 8, 494. [CrossRef] [PubMed]

33. Buettner, C.; Noll, M. Differences in microbial key players in anaerobic degradation between biogas and sewage treatment plants. Int. Biodeterior. Biodegrad. 2018, 133, 124-132. [CrossRef]

34. Bushnell, B.; Rood, J.; Singer, E. BBMerge-Accurate paired shotgun read merging via overlap. PLoS ONE 2017, 12, e0185056. [CrossRef]

35. Schloss, P.D.; Westcott, S.L.; Ryabin, T.; Hall, J.R.; Hartmann, M.; Hollister, E.B.; Lesniewski, R.A.; Oakley, B.B.; Parks, D.H.; Robinson, C.J.; et al. Introducing mothur: Open-source, platform-independent, community-supported software for describing and comparing microbial communities. Appl. Environ. Microbiol. 2009, 75, 7537-7541. [CrossRef]

36. Quast, C.; Pruesse, E.; Yilmaz, P.; Gerken, J.; Schweer, T.; Yarza, P.; Peplies, J.; Glöckner, F.O. The SILVA ribosomal RNA gene database project: Improved data processing and web-based tools. Nucleic Acids Res. 2013, 41, D590-D596. [CrossRef] 
37. Oksanen, J.F.; Blanchet, G.; Friendly, M.; Kindt, R.; Legendre, P.; McGlinn, D.; Minchin, P.R.; O’Hara, R.B.; Simpson, G.L.; Solymos, P.; et al. Package "vegan": Community Ecology Package. Available online: https://github.com/vegandevs/vegan (accessed on 20 February 2021).

38. Royston, P. Approximating the Shapiro-Wilk W-test for non-normality. Stat. Comput. 1992, 2, 117-119. [CrossRef]

39. Faust, K.; Raes, J. CoNet app: Inference of biological association networks using Cytoscape. F1000Res 2016, 5, 1519. [CrossRef] [PubMed]

40. Shannon, P.; Markiel, A.; Ozier, O.; Baliga, N.S.; Wang, J.T.; Ramage, D.; Amin, N.; Schwikowski, B.; Ideker, T. Cytoscape: A software environment for integrated models of biomolecular interaction networks. Genome Res. 2003, 13, 2498-2504. [CrossRef]

41. Brown, M.B. 400: A method for combining non-independent, one-sided tests of significance. Biometrics 1975, 31, 987. [CrossRef]

42. Benjamini, Y.; Hochberg, Y. Controlling the false discovery rate: A practical and powerful approach to multiple testing. J. R. Stat. Soc. Series B (Methodological) 1995, 57, 289-300. [CrossRef]

43. Su, G.; Kuchinsky, A.; Morris, J.H.; States, D.J.; Meng, F. GLay: Community structure analysis of biological networks. Bioinformatics 2010, 26, 3135-3137. [CrossRef]

44. Guimerà, R.; Nunes Amaral, L.A. Functional cartography of complex metabolic networks. Nature 2005, 433, 895-900. [CrossRef] [PubMed]

45. Neves Forte, W.C.; Mayumi Sumita, J.; Gianini Rodrigues, A.; Liuson, D.; Tanaka, E. Rebound phenomenon to systemic corticosteroid in atopic dermatitis. Allergol. Immunopathol. 2005, 33, 307-311. [CrossRef]

46. Geoghegan, J.A.; Irvine, A.D.; Foster, T.J. Staphylococcus aureus and atopic dermatitis: A complex and evolving relationship. Trends Microbiol. 2018, 26, 484-497. [CrossRef]

47. Byrd, A.L.; Belkaid, Y.; Segre, J.A. The human skin microbiome. Nat. Rev. Microbiol. 2018, 16, 143-155. [CrossRef]

48. Zeeuwen, P.L.J.M.; Boekhorst, J.; Ederveen, T.H.A.; Kleerebezem, M.; Schalkwijk, J.; van Hijum, S.A.F.T.; Timmerman, H.M. Reply to Meisel et al. J. Investig. Dermatol. 2017, 137, 961-962. [CrossRef]

49. Nizioł-Łukaszewska, Z.; Bujak, T.; Wasilewski, T.; Szmuc, E. Inulin as an effectiveness and safe ingredient in cosmetics. Polish J. Chem. Technol. 2019, 21, 44-49. [CrossRef]

50. Parvez, S.; Malik, K.A.; Ah Kang, S.; Kim, H.-Y. Probiotics and their fermented food products are beneficial for health. J. Appl. Microbiol. 2006, 100, 1171-1185. [CrossRef]

51. Yildirim, Z.; Winters, D.K.; Johnson, M.G. Purification, amino acid sequence and mode of action of bifidocin B produced by Bifidobacterium bifidum NCFB 1454. J. Appl. Microbiol. 1999, 86, 45-54. [CrossRef] [PubMed]

52. Guo, X.; Chen, J.; Sun, H.; Luo, L.; Gu, Y.; Yi, Y.; Wang, X.; Shan, Y.; Liu, B.; Zhou, Y.; et al. Mining, heterologous expression, purification and characterization of 14 novel bacteriocins from Lactobacillus rhamnosus LS-8. Int. J. Biol. Macromol. 2020, 164, 2162-2176. [CrossRef]

53. Diep, D.B.; Håvarstein, L.S.; Nes, I.F. Characterization of the locus responsible for the bacteriocin production in Lactobacillus plantarum C11. J. Bacteriol. 1996, 178, 4472-4483. [CrossRef]

54. Kawai, Y.; Saito, T.; Kitazawa, H.; Itoh, T. Gassericin A; an uncommon cyclic bacteriocin produced by Lactobacillus gasseri LA39 linked at N- and C-terminal ends. Biosci. Biotechnol. Biochem. 1998, 62, 2438-2440. [CrossRef] [PubMed]

55. Rammelsberg, M.; Mller, E.; Radler, F. Caseicin 80: Purification and characterization of a new bacteriocin from Lactobacillus casei. Arch. Microbiol. 1990, 154, 249-252. [CrossRef]

56. Park, S. Potentiation of hydrogen peroxide, nitric oxide, and cytokine production in RAW 264.7 macrophage cells exposed to human and commercial isolates of Bifidobacterium. Int. J. Food Microbiol. 1999, 46, 231-241. [CrossRef]

57. Baurecht, H.; Rühlemann, M.C.; Rodríguez, E.; Thielking, F.; Harder, I.; Erkens, A.-S.; Stölzl, D.; Ellinghaus, E.; Hotze, M.; Lieb, W.; et al. Epidermal lipid composition, barrier integrity, and eczematous inflammation are associated with skin microbiome configuration. J. Allergy Clin. Immunol. 2018, 141, 1668-1676.e16. [CrossRef]

58. Kwaszewska, A.; Sobiś-Glinkowska, M.; Szewczyk, E.M. Cohabitation-relationships of corynebacteria and staphylococci on human skin. Folia Microbiol. (Praha) 2014, 59, 495-502. [CrossRef]

59. Talento, A.F.; Malnick, H.; Cotter, M.; Brady, A.; McGowan, D.; Smyth, E.; Fitzpatrick, F. Brevibacterium otitidis: An elusive cause of neurosurgical infection. J. Med. Microbiol. 2013, 62, 486-488. [CrossRef]

60. Gong, J.Q.; Lin, L.; Lin, T.; Hao, F.; Zeng, F.Q.; Bi, Z.G.; Yi, D.; Zhao, B. Skin colonization by Staphylococcus aureus in patients with eczema and atopic dermatitis and relevant combined topical therapy: A double-blind multicentre randomized controlled trial. Br. J. Dermatol. 2006, 155, 680-687. [CrossRef] [PubMed] 